\title{
Pairing and Condensation of Laughlin Quasiparticles in Fractional Quantum Hall Systems
}

\author{
A. WóJS ${ }^{a, b, *}$, KYUNG-SoO Yi ${ }^{b, c}$ AND J.J. QUINN ${ }^{b}$ \\ ${ }^{a}$ Wrocław University of Technology \\ Wybrzeże Wyspiańskiego 27, 50-370 Wrocław, Poland \\ ${ }^{b}$ University of Tennessee, Knoxville, Tennessee 37996, USA \\ ${ }^{c}$ Pusan National University, Pusan 609-735, Korea
}

\begin{abstract}
From the analysis of their interaction pseudopotentials, it is argued that (at certain filling factors) Laughlin quasiparticles can form pairs. It is further proposed that such pairs could have Laughlin correlations with one another and form condensed states of a new type. The sequence of fractions corresponding to these states includes all new fractions observed recently in experiment (e.g., $\nu=5 / 13,3 / 8$, or $4 / 11$ ).
\end{abstract}

PACS numbers: 71.10.Pm, 73.43.-f

\section{Introduction}

In a recent experiment Pan et al. [1] observed the fractional quantum Hall (FQH) effect $[2,3]$ at novel filling fractions $\nu$ of the lowest Landau level (LL). Among other features, the FQH effect is a macroscopic manifestation of an incompressible character of the many-body ground state formed at a specific filling $\nu$. The new spin-polarized FQH states occur at filling factors outside the Jain sequence [4] of composite fermion (CF) states. Some of them, as $\nu=4 / 11$ or $4 / 13$, appear in the Haldane hierarchy [5] of quasiparticle (QP) condensates, and therefore at first sight it might only be surprising that they have not been observed earlier. However, the "hierarchical" interpretation of these states was questioned [6] because of the specific form of the QP-QP interaction, which leaves their interpretation quite uncertain. Others, such as the $\nu=3 / 8$ or $3 / 10$ states, do not belong to the Haldane hierarchy, and thus the origin of their incompressibility is puzzling in an even more obvious way. In the CF picture, these even-denominator

*corresponding author; e-mail: arkadiusz.wojs@pwr.wroc.pl 
fractions correspond to the half-filled first excited $(n=1)$ CF LL. While for the electrons half-filling their $n=1$ LL (including the double degeneracy of the lowest LL, this amounts to the total electron filling of $\nu=2+1 / 2=5 / 2$ ) the FQH effect has been known for quite some time [7] (the incompressible $\nu=5 / 2$ state is known as a Moore-Read state [8]), the similar behavior of electrons and CF's at this filling is rather unexpected. Pan et al. take their observations as evidence for residual CF-CF interactions, sufficiently strong to cause emergence of new FQH states (that, unlike virtually all observed fractions, cannot be predicted from the noninteracting CF model or standard Haldane hierarchy). However, they ignore the theoretical investigations in which these interactions were studied in detail $[6,9,10]$.

In this paper we propose an explanation for these new states involving the formation of QP pairs which display Laughlin correlations with one another. First, we explain the connection between the CF model and the QP hierarchy $[5,6,9]$. Second, we recall two simple types of two-body correlations, Laughlin correlations and pairing, that may occur in an interacting system depending on the filling factor $\nu$ and on whether $V(\mathcal{R})$ is super- or subharmonic at the relevant range [11]. Then, knowing the QP-QP pseudopotential $V_{\mathrm{QP}}(\mathcal{R})$, we apply the concept of Laughlin condensed states of (bosonic) pairs (used earlier for the electrons in the $n=1 \mathrm{LL}$ to describe such FQH states as $\nu=5 / 2$ or $7 / 3$ [12]) to the particles or holes in a partially filled CF LL, i.e., to Laughlin quasielectrons (QE's) or quasiholes (QH's). Finally, we propose the novel hierarchy of FQH states in which the incompressibility results from the condensation of QP pairs ( $\mathrm{QE}_{2}$ 's or $\mathrm{QH}_{2}$ 's) into Laughlin correlated pair states. The series of FQH states derived from the parent $\nu=1 / 3$ state include all novel fractions: $\nu=5 / 13,3 / 8,4 / 11$, and $6 / 17$ for the QE's and $\nu=5 / 17,3 / 10,4 / 13$, and $6 / 19$ for the QH's.

\section{Laughlin QP's and CF's}

Let us begin with recalling the connection between Laughlin QP's [3] and the CF model (equivalent to the mean-field Chern-Simons transformation) [4]. Laughlin QP's are the actual elementary excitations of a two-dimensional electron liquid filling a fraction of the lowest LL. They have well-defined and known wave functions (and thus also density profiles, size, etc.) and single-particle energy. They carry (fractional) electric charge, counted with respect to the uniformly spread charge of the underlying Laughlin state. The negatively and positively charged QP's are called quasielectrons (QE's) and quasiholes (QH's), respectively. Moving in the external magnetic field, both kinds of QP's follow cyclotron orbits, conveniently labeled by angular momentum.

Since the uniform-density Laughlin states only occur at a discrete series of filling factors $(\nu=1 / 3,1 / 5, \ldots)$, and Laughlin QP's are simply the least-energy excitations able to carry charge in excess of the value corresponding to the nearest 
Laughlin state, their type and number depend on $\nu$. At $\nu$ precisely equal to any of the Laughlin values $\nu_{p}=(2 p+1)^{-1}$ no QP's exist in the ground state and they may only appear in a form of excitonic, charge-neutral QE-QH pairs. At $\nu$ slightly smaller (or larger) than $\nu_{p}$, a number $N_{\mathrm{QP}}$ of the " $p$-type" QE's (or QH's) appear in the Laughlin liquid. The QP number is equal to the difference between the total magnetic flux through the sample and its value corresponding to the nearest "parent" Laughlin state, measured in units of elementary flux, $N_{\mathrm{QP}}=\left|\Phi-\Phi_{p}\right| / \phi_{0}$.

For electrons, the filling factor is expressed through the flux $\Phi$ and the electron number $N$ as $\nu=\Phi /\left(N \phi_{0}\right)$, For the QP's of the parent $\nu_{p}$ Laughlin state, each carrying charge $\pm e /(2 p+1)$, the LL degeneracy is reduced by a factor of $(2 p+1)$ compared to the electron value. Consequently, the QP filling factor is equal to $\nu_{\mathrm{QP}}=\Phi /\left[(2 p+1) N_{\mathrm{QP}} \phi_{0}\right]$. Note that $\nu_{\mathrm{QP}}$ is a linear function of $\nu$ near each value of $\nu_{p}$, The situation with $\nu$ far away from the nearest $\nu_{p}$, corresponds to a large filling factor for appropriate QP's whose mutual interactions and filling of higher QP LL's both become important.

In the mean-field CF picture, the reduction of the QP LL degeneracy is attributed to a reduced magnetic field $B^{*}=B /(2 p+1)$ rather than to a reduced QP charge. In the simplest formulation of the model, the effective field $B^{*}$ results from the capture of an even number of magnetic flux quanta $2 p \phi_{0}$ by each electron (such bound state is called a CF), and the QE's and QH's are pictured as particles and vacancies in the otherwise empty (full) CF LL's.

The CF picture turns out very useful for the description of many properties of the QP's (e.g., size of cyclotron orbits or LL degeneracy) or of the FQH systems in general (e.g., the values of $\nu$ at which the incompressibility results from a complete filling of the QP/CF LL's). However, the QP-QP interactions important for the present problem in the CF model arise as a combination of rather complicated twoand three-body gauge interactions between charges and fluxes, and because of this difficulty, are usually neglected. It is therefore important to realize that the QP-QP interaction really is a Coulomb interaction between a pair of charged particles. For example, for two identical QP's it is repulsive, and at long range it is similar to the electron-electron repulsion, only reduced in magnitude by $(2 p+1)^{2}$ because of a smaller QP charge. On the other hand, the exact form of the QP-QP interaction at short range (where it is different from the electron-electron repulsion because of the particular QP charge-density profile) has been quite accurately calculated numerically [6].

\section{Haldane hierarchy and Jain sequence}

Knowing that the $\mathrm{QE}-\mathrm{QE}$ and $\mathrm{QH}-\mathrm{QH}$ interactions are generally repulsive, Haldane proposed [5] condensation of QE's and QH's into the hierarchy of "daughter" states at the series of Laughlin values of $\nu_{\mathrm{QE}}$ or $\nu_{\mathrm{QH}}$. In these states, the appropriate QP's correlate with one another in the same way as the electrons do in the Laughlin states, and their elementary excitations are simply a new gen- 
eration of QP's. Assuming such QP condensation at each level of the hierarchy one would predict incompressibility of the whole electron system at all filling factors given by any odd-denominator fraction. This is in striking disagreement with the experiments, and the reason for this discrepancy is that although Coulombic, the $\mathrm{QP}-\mathrm{QP}$ interaction at short range is not quite identical to the electron-electron repulsion responsible for the Laughlin correlations. As a result, the QP's form Laughlin liquids only at very few of the Laughlin fractions, which eliminates all but a few valid "hierarchy" fractions [6], in good agreement with the experiment. The same series of fractions arise naturally in the CF picture. These are the states at $\nu=(2 p+1 / n)^{-1}$, corresponding to a number $n$ of completely filled CF LL's each carrying flux $2 p \phi_{0}$.

The new FQH states [1] occur at the values of $\nu$ from outside the Jain sequence, and thus corresponding to only partial filling of a CF LL. Hence, in the $\mathrm{CF}$ picture, their incompressibility implies role of $\mathrm{CF}-\mathrm{CF}$ interactions. In the QP hierarchy picture, the new states either coincide with the "invalid" fractions (e.g., $\nu=4 / 11$ ) or are new fractions altogether (e.g., $\nu=3 / 8$ ). In both cases it is clear that the origin of observed incompressibility lies in the special form of QP-QP correlations, and that these correlations are of a new (non-Laughlin) type.

\section{QP-QP pseudopotential}

The nature of QP correlations depends critically on the form of pseudopotential $V_{\mathrm{QP}}(\mathcal{R})$ describing their pair interaction energy $V_{\mathrm{QP}}$ as a function of relative pair angular momentum $\mathcal{R}$. We have shown earlier $[11,12]$ that the correlations are of the Laughlin type (i.e., the particles tend to avoid pair states with one or more of the smallest values of $\mathcal{R}=1,3, \ldots)$ only if $V(\mathcal{R})$ is "superharmonic" at the relevant values of $\mathcal{R}$ for a given filling factor $\nu$ (specifically, at $\mathcal{R}=2 p-1$ for $\nu \sim(2 p+1)^{-1}$, where $\left.p=1,2, \ldots\right)$. Laughlin correlations defined in this way justify reapplication of the CF picture to the QP's to select the lowest states of the whole many-body spectrum, and lead to the incompressible QP "daughter" states of the standard CF hierarchy [9]. The superharmonic repulsion is defined as one for which $V$ decreases more quickly than linearly as a function of the average particle-particle separation $\left\langle r^{2}\right\rangle$ for the consecutive pair eigenstates labeled by $\mathcal{R}$. In spherical geometry [5], most convenient for finite-size calculations, this means that $V$ increases more quickly than linearly as a function of $L(L+1)$, i.e., of the squared total pair angular momentum $L=2 l-\mathcal{R}$, where $l$ is the single-particle angular momentum.

The qualitative behavior of the $\mathrm{QP}-\mathrm{QP}$ interaction pseudopotential $V_{\mathrm{QP}}(\mathcal{R})$ at short range is well known from numerical studies of small systems $[6,9,10]$. On the other hand, the repulsive character of the QP-QP interaction and the long-range behavior of $V_{\mathrm{QP}}(\mathcal{R}) \sim \mathcal{R}^{-1 / 2}$ follow from the fact that QP's are charged particles (the form of QP charge density affects $V_{\mathrm{QP}}$ only at short range, comparable to the QP size). Combining the above arguments, it is clear that the dominant 
features of $V_{\mathrm{QE}}$ are the small value at $\mathcal{R}=1$ and a strong maximum at $\mathcal{R}=3$. Analogous analysis for the QH's yields maxima at $\mathcal{R}=1$ and 5 , and nearly vanishing $V_{\mathrm{QH}}(3)$.

\section{QP pairing}

It is evident that $V_{\mathrm{QE}}$ does not support Laughlin QE-QE correlations. Instead, we expect that at least some of the QE's will form pairs $\left(\mathrm{QE}_{2}\right)$ at $\mathcal{R}=1$. A paired state would be characterized by a greatly reduced fractional parentage $\mathcal{G}$ [11] from the strongly repulsive $\mathcal{R}=3$ state compared to the Laughlin correlated state, and have the lower total interaction energy $E=\frac{1}{2} N(N-1) \sum_{\mathcal{R}} \mathcal{G}(\mathcal{R}) V(\mathcal{R})$. Let us stress that such pairing is not a result of some attractive QE-QE interaction, but due to a tendency to a void the most strongly repulsive $\mathcal{R}=3$ pair state. At sufficiently high QE density this can only be achieved by having significant $\mathcal{G}(1)$, which can be interpreted as pairing into the $\mathrm{QE}_{2}$ molecules. By analogy, the $\mathrm{QH}$ pairing is expected at $\mathcal{R}=3$. The range of $\nu_{\mathrm{QP}}$ at which pairing can be considered is limited by the condition that the separation between the pairs must exceed the pair size. While for the QE pairs this is satisfied at any $\nu_{\mathrm{QE}}<1$, the QH pairing can only occur at $\nu_{\mathrm{QH}}<1 / 3$.

\section{Laughlin correlations between pairs}

Having established that the QP fluid consists of (bosonic) $\mathrm{QP}_{2}$ molecules, the $\mathrm{QP}_{2}-\mathrm{QP}_{2}$ interactions need be studied to understand correlations. The $\mathrm{QP}_{2}-\mathrm{QP}_{2}$ interaction is described by an effective pseudopotential $V_{\mathrm{QP}_{2}}(\mathcal{R})$ that includes correlation effects caused by the fact that the two-pair wave function must be symmetric under exchange of the whole $\mathrm{QP}_{2}$ bosons and at the same time antisymmetric under exchange of any pair of the QP fermions. This problem is analogous to that of interaction between the electron pairs in the $n=1 \mathrm{LL}[12]$.

Although we do not know $V_{\mathrm{QP}_{2}}(\mathcal{R})$ accurately, we expect that since it is due to the repulsion between the $\mathrm{QP}$ 's that belong to different $\mathrm{QP}_{2}$ pairs, it might be superharmonic at the range corresponding to the $\mathrm{QP}_{2}-\mathrm{QP}_{2}$ separation. Our preliminary numerical results for four QE's seem to support this idea. However, in contrast to the $n=1$ electron LL [12], the lack of accurate data for $V_{\mathrm{QP}}$ at the intermediate range makes such calculations uncertain.

\section{Condensed pair states}

The assumption of Laughlin correlations between the $\mathrm{QP}_{2}$ bosons implies the sequence of Laughlin condensed $\mathrm{QP}_{2}$ states that can be conveniently described using the "composite boson" (CB) model [12]. Let us use spherical geometry and consider the system of $N_{1}$ fermions (QP's) each with (integral or half-integral) 
angular momentum $l_{1}$ (i.e., in a $L \mathrm{~L}$ of degeneracy $g_{1}=2 l_{1}+1$ ). Neglecting the finite-size corrections, this corresponds to the filling factor $\nu_{1}=N_{1} / g_{1}$. Let the fermions form $N_{2}=\frac{1}{2} N_{1}$ bosonic pairs each with angular momentum $l_{2}=2 l_{1}-\mathcal{R}_{1}$, where $\mathcal{R}_{1}$ is an odd integer. The filling factor for the system of pairs, defined as $\nu_{2}=N_{2} / g_{2}$ where $g_{2}=2 l_{2}+1$, equals to $\nu_{2}=\frac{1}{4} \nu_{1}$. The allowed states of two bosonic pairs are labeled by total angular momentum $L=2 l_{2}-\mathcal{R}_{2}$, where $\mathcal{R}_{2}$ is an even integer. Of all even values of $\mathcal{R}_{2}$, the lowest few are not allowed because of the Pauli exclusion principle applied to the individual fermions. The condition that the two-fermion states with relative angular momentum smaller than $\mathcal{R}_{1}$ are forbidden, is equivalent to the elimination of the states with $\mathcal{R}_{2} \leq 4 \mathcal{R}_{1}$ from the two-boson Hilbert space. Such a "hard core" can be accounted for by a CB transformation with $4 \mathcal{R}_{1}$ flux quanta attached to each boson [13]. This gives effective CB angular momentum $l_{2}^{*}=l_{2}-2 \mathcal{R}_{1}\left(N_{2}-1\right)$, LL degeneracy $g_{2}^{*}=g_{2}-4 \mathcal{R}_{1}\left(N_{2}-1\right)$, and filling factor $\nu_{2}^{*}=\left(\nu_{2}^{-1}-4 \mathcal{R}_{1}\right)^{-1}$.

The CB's defined in this way condense into their only allowed $l_{2}^{*}=0$ state $\left(\nu_{2}^{*}=\infty\right)$ when the corresponding fermion system has the maximum density at which pairing is still possible, $\nu_{1}=\mathcal{R}_{1}^{-1}$. At lower filling factors, the CB LL is degenerate and the spectrum of all allowed states of the $N_{2}$ CB's represents the spectrum of the corresponding paired fermion system. In particular, using the assumption of the superharmonic form of boson-boson repulsion, condensed CB states are expected at a series of Laughlin filling factors $\nu_{2}^{*}=(2 q)^{-1}$. Here, $2 q$ is an even integer corresponding to the number of additional magnetic flux quanta attached to each $\mathrm{CB}$ in a subsequent CB transformation, $l_{2}^{*} \rightarrow l_{2}^{* *}=l_{2}^{*}-q\left(N_{2}-1\right)$, to describe Laughlin correlations between the original CB's of angular momentum $l_{2}^{*}$. From the relation between the fermion and CB filling factors, $\nu_{1}^{-1}=\left(4 \nu_{2}^{*}\right)^{-1}+\mathcal{R}_{1}$, we find the following sequence of fractions corresponding to the Laughlin condensed pair states, $\nu_{1}^{-1}=q / 2+\mathcal{R}_{1}$. Finally, we set $\mathcal{R}_{1}=1$ for the QE's and $\mathcal{R}_{1}=3$ for the QH's, and use the hierarchy equation [6], $\nu^{-1}=2 p+\left(1 \pm \nu_{\mathrm{QP}}\right)^{-1}$, to calculate the following sequences of electron filling factors, $\nu$, derived from the parent $\nu=(2 p+1)^{-1}$ state

$$
\nu^{-1}=2 p+1 \mp(2+q / 2)^{-1}
$$

where "+" corresponds to the QE's and "-" to the QH's. Remarkably, all the fractions reported by Pan et al. are among those predicted for the $\nu=1 / 3$ parent. Note also that the same values of $q=1,2,4$, and 8 describe both observed $\mathrm{QE}$ and QH states. This indicates similarity of the QE-QE and QH-QH pseudopotentials and suggests that both $V_{\mathrm{QE}_{2}}$ and $V_{\mathrm{QH}_{2}}$ may be superharmonic only at the corresponding four values of $\mathcal{R}$ (in such case, the remaining fractions could not be observed even in most ideal samples).

\section{Conclusion}

We have studied the QP-QP interactions leading to novel spin-polarized FQH states in the lowest LL. Using the knowledge of QP-QP pseudopotentials 
and a general dependence of the form of correlations on the super- or subharmonic behavior of the pseudopotential, we have shown that QP's form pairs over a certain range of filling factor $\nu_{\mathrm{QP}}$. Then, we argued that the correlations between the QP pairs should be of Laughlin type and proposed a hierarchy of condensed paired QP states. The proposed hierarchy of fractions agrees remarkably well with the recent experiment of Pan et al. [1].

\section{Acknowledgments}

The authors thank Jennifer J. Quinn and Josef Tobiska for helpful discussions and acknowledge support from US DoE Grant DE-FG 02-97ER45657. A.W. acknowledges support from the State Committee for Scientific Research grant 2P03B02424. K.S.Y. acknowledges support from KOSEF grant R14-2002-029-01002-0.

\section{References}

[1] W. Pan, H.L. Störmer, D.C. Tsui, L.N. Pfeiffer, K.W. Baldwin, K.W. West, Phys. Rev. Lett. 90, 016801 (2003).

[2] D.C. Tsui, H.L. Störmer, A.C. Gossard, Phys. Rev. Lett. 48, 1559 (1982).

[3] R. Laughlin, Phys. Rev. Lett. 50, 1395 (1983).

[4] J.K. Jain, Phys. Rev. Lett. 63, 199 (1989).

[5] F.D.M. Haldane, Phys. Rev. Lett. 51, 605 (1983).

[6] A. Wójs, J.J. Quinn, Phys. Rev. B 61, 2846 (2000).

[7] R.L. Willet, J.P. Eisenstein, H.L. Störmer, D.C. Tsui, A.C. Gossard, J.H. English, Phys. Rev. Lett. 59, 1776 (1987).

[8] G. Moore, N. Read, Nucl. Phys. B 360, 362 (1991).

[9] P. Sitko, S.N. Yi, K.-S. Yi, J.J. Quinn, Phys. Rev. Lett. 76, 3396 (1996); P. Sitko, K.-S. Yi, J.J. Quinn, Phys. Rev. B 56, 12417 (1997).

[10] S.-Y. Lee, V.W. Scarola, J.K. Jain, Phys. Rev. Lett. 87, 256803 (2001).

[11] A. Wójs, J.J. Quinn, Philos. Mag. B 80, 1405 (1900); Acta Phys. Pol. A 96, 593 (1999); J.J. Quinn, A. Wójs, J. Phys., Condens. Matter 12, R265 (2000).

[12] A. Wójs, Phys. Rev. B 63, 125312 (2001); A. Wójs, J.J. Quinn, Physica E 12, 63 (2002).

[13] J.J. Quinn, J. Tobiska, unpublished; A.T. Benjamin, J.J. Quinn, A. Wójs, J. Combinat. Theory A 95, 390 (2001). 